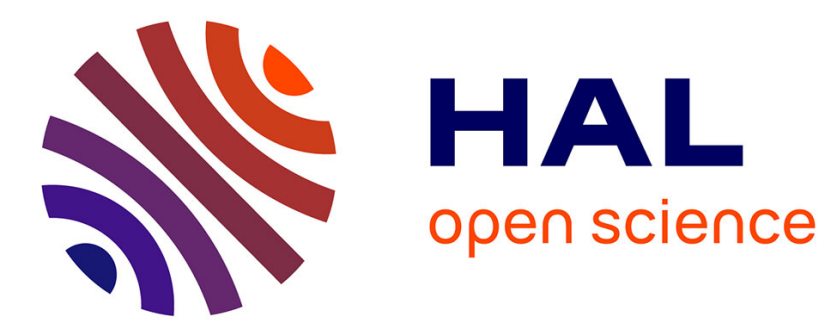

\title{
Multiple-antenna Transmitted-Reference communication systems performance in presence of narrowband interference
}

\author{
Pascal Chargé, Guillaume Fumat, Pascal Acco
}

\section{To cite this version:}

Pascal Chargé, Guillaume Fumat, Pascal Acco. Multiple-antenna Transmitted-Reference communication systems performance in presence of narrowband interference. IEEE 11th International Workshop on Signal Processing Advances in Wireless Communications (SPAWC 2010), Jun 2010, Marrakech, France. 10.1109/SPAWC.2010.5671000 . hal-01886033

\section{HAL Id: hal-01886033 \\ https://hal.laas.fr/hal-01886033}

Submitted on 3 May 2019

HAL is a multi-disciplinary open access archive for the deposit and dissemination of scientific research documents, whether they are published or not. The documents may come from teaching and research institutions in France or abroad, or from public or private research centers.
L'archive ouverte pluridisciplinaire HAL, est destinée au dépôt et à la diffusion de documents scientifiques de niveau recherche, publiés ou non, émanant des établissements d'enseignement et de recherche français ou étrangers, des laboratoires publics ou privés. 


\title{
Multiple-antenna Transmitted-Reference communication systems performance in
}

\author{
presence of narrowband interference
}

\author{
Pascal ChARgé, Guillaume Fumat, Pascal ACCO \\ LATTIS-INSA, Université de Toulouse \\ pascal.charge@insa-toulouse.fr
}

\begin{abstract}
The aim of this paper is to show the improvement of Transmitted-Reference (TR) communication systems when an antenna array is used at the receiver side. A generalized model of communication waveforms is involved so that this study is valid for any kind of carrier waveforms, as well as sinusoidal signals as in conventional narrowband communications, UWB impulses or even chaotic waveforms. Thus a BER performance expression of such a TR-based system is provided in presence of a narrowband interferer.
\end{abstract}

\section{INTRODUCTION}

The typical bandwidth of a UWB signal is a few $\mathrm{GHz}$, and the carriers may be either fixed waveforms as in UWB impulse radio or continuously varying wideband chaotic signals. Due to their low power spectral density, the UWB carriers allow the reuse of already occupied frequency bands, but on the other hand the detection process becomes highly challenging. Among different possibilities, Transmitted-Reference (TR) modulation techniques are very good candidates to cope with such communication channels. Many proposed works on this subject can be found in the very recent literature [1][2][3][4]. BER performance of such TR-based systems has been studied as in [5][6] in the case of chaotic waveforms or in [4][7] with impulse radio carriers. These studies show limitations of TR schemes mainly because the detectors have to deal with very low signal power spectral density with relatively high noise and, interference power cannot be ignored. In order to improve the performance of such systems, noise rejection techniques can be performed but unfortunately, even computationally intensive noise cleaning algorithms are not efficient when the signal to noise ratio reaches that of a real radio application.

Another solution, which is investigated here, instead of reducing the noise, is to increase the signal power at the receiver side. This can be done by using multiple-antenna receivers. A delay-sum antenna array autocorrelation receiver has been proposed recently in [8] and [9]. By exploiting the spatial diversity provided by an antenna array, the signal-tonoise power ratio (SNR) at the receiver can be drastically improved, even in the case of UWB signals. The purpose of this work is then to investigate the improvement of the performance of wideband TR-based autocorrelation systems when an antenna array is used at the receivers, and when a high power narrowband interferer (NBI) lies in the operating frequency band. In this paper, by assuming that the Direction of Arrival (DOA) of the wideband signal of interest (WSOI) has been estimated or is known a priori, a delay-and-sum beamforming at the output of the antenna array is processed.
Then a classical correlator-based detector is involved to demodulate the data information. According to the generalized communication waveforms model [10][11], a wideband signal model is proposed which is valid for any kind of carrier waveforms, as well as sinusoidal signals, UWB impulses or chaotic waveforms. Thus a BER performance expression of such a TR-based system is derived, which depends on both the signal bandwidth and power, and the antenna array features. Finally, numerical simulations are performed to emphasize that the use of antenna array can drastically improve BER performance, and can also significantly reduce interference caused by an undesired high power signal impinging on the receiver's array.

\section{TRANSMITTED-REFERENCE ANTENNA ARRAY RECEIVER}

In order to investigate performance improvements provided by an antenna array at the receiver side, let consider the receiver architecture in figure 1 . An array of antennas collects both signals and noise with space diversity. Assuming that the Direction-of-Arrival (DOA) of the signal of interest is known, a delay-and-sum beamforming at the output of the antenna array is processed. Then a classical correlator-based detector is involved to demodulate the data information.

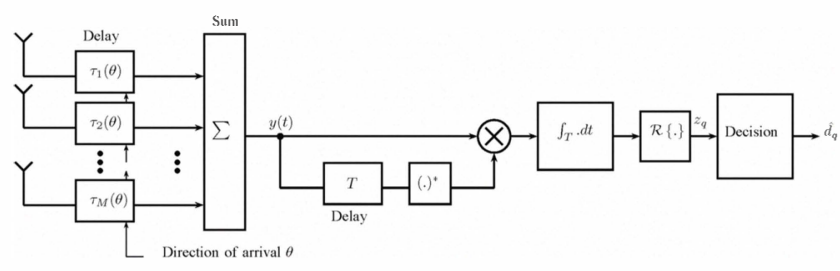

Figure 1. Multiple-antenna receiver and correlation-based detector

\section{A. Antenna array}

An antenna array consists of multiple sensors which are located at different points in space. Each sensor receives incoming signals delayed differently, depending on the array geometry. Each sensor also collects noise but, this additive noise is usually assumed to be uncorrelated with the incoming signals. In this work, without loss of generality, we will consider the case of an array of $M$ isotropic antennas. When the signal of interest $s(t)$ is impinging on the array with a given DOA $\theta$ (measured from the array broadside), the 
measurement at the output of the $m^{t h}(m=1, \ldots, M)$ sensor can be described by

$$
z_{m}(t)=s\left(t-\tau_{m}(\theta)\right)+n_{m}(t)
$$

where $\tau_{m}(\theta)$ denotes the relative time delay at the $m^{t h}$ antenna, and $n_{m}(t)$ is the noise contribution.

\section{B. Delay-and-sum signal}

We are interested here in the general framework of Transmitted-Reference (TR) radio systems. In such a scheme the symbol duration is divided into two time slots, a reference signal time slot and an information-bearing signal time slot. This information bearing signal is in fact the reference signal multiplied by the binary data to be transmitted.

Assuming that the two above mentioned time slots have the same duration $T$, the whole symbol duration is $2 T$. Then the harmonic form of the Fourier series representation in the generalized wideband communication waveforms model [10] is applied on the reference signal only. Moreover, the received signal is assumed to be a wideband signal, bandlimited by the bandpass channel filter of bandwidth $W$. Spectral components of this wideband signal lie inside the frequency range $K_{1} / T \leq|f| \leq K_{2} / T$, where $K_{1}$ and $K_{2}$ are positive integers. It comes that $K_{2}-K_{1}+1=W T$. Let define the set of integers $\mathcal{K}=\left\{K_{1}, K_{1}+1 \ldots, K_{2}\right\}$. Then a complex representation of the wideband received signal using complex envelopes, at the $m^{t h}$ antenna of the array is given by

$z_{m}(t)=\sum_{q=0}^{\infty} \sum_{k \in \mathcal{K}} \rho_{k}^{q} g_{d_{q}}\left(t+\tau_{m}(\theta)-q 2 T\right) e^{j \omega k\left(t+\tau_{m}(\theta)\right)}+b_{m}(t)$

where $\omega=\frac{2 \pi}{T}$ and $\rho_{k}^{q}$ is the complex Fourier coefficient (collecting the harmonic amplitude and the phase angle) of the $k^{\text {th }}$ harmonic component of the reference signal at the $q^{\text {th }}$ symbol time slot of the wideband signal of interest (WSOI). $d_{q} \in\{+1,-1\}$ is the $q^{\text {th }}$ binary data information to be transmitted, and $g_{d_{q}}(t)$ is the TR symbol pattern given by

$$
g_{d_{q}}(t)= \begin{cases}1 & \text { if } 0 \leq t<T \\ d_{q} & \text { if } T \leq t<2 T \\ 0 & \text { elsewhere }\end{cases}
$$

Whatever the shape of the waveforms (deterministic pulses, pseudo-random or chaotic sequences) some variations (timehopping or polarity sequences) are often introduced at the emitter and the channel also brings some time-varying distortions. Hence it is reasonable to assume that the received signal is random. Each coefficient $\rho_{k}^{q}$ in (2) will be considered as a zero-mean complex circular random variable. Power spectral density of the WSOI is assumed to be constant in the whole bandwidth of the signal, and is equal to

$$
\mathrm{E}\left[\left|\rho_{k}^{q}\right|^{2}\right]=\sigma_{\rho}^{2}, \forall(k, q)
$$

The mean-power of the WSOI is then equal to $W \sigma_{\rho}^{2}$, and the average-bit-energy of the WSOI is

$$
E_{b}=2 W T \sigma_{\rho}^{2}
$$

In equation (2), the term $b_{m}(t)$ collects both an additive complex circular Gaussian noise and interferer signals. These interferer signals can be narrowband interferer (NBI) signals or also wideband interferer (WBI) signals. Then assuming that the direction of arrival $\theta$ of the WSOI is known, the delayand-sum output signal is

$y(t)=M \sum_{q=0}^{\infty} \sum_{k \in \mathcal{K}} \rho_{k}^{q} g_{d_{q}}(t-2 q T) e^{j \omega k t}+\sum_{m=1}^{M} b_{m}\left(t-\tau_{m}(\theta)\right)$

\section{Correlation-based demodulation}

This signal (6) is entering into the correlation-based demodulator as shown in figure 1. Assuming that the symbol time synchronization is achieved, the decision variable associated to the $q^{t h}$ transmitted symbol, at the input of the decision circuit is

$$
z_{q}=\mathcal{R}\left\{\int_{(2 q+1) T}^{2(q+1) T} y(t) y^{*}(t-T) d t\right\}
$$

where $\mathcal{R}\{x\}$ denotes the real part of $x$ and (.)* is the complex conjugate operator. From (6) it is straightforward to express this decision variable under the following global form

$$
z_{q}=d_{q} M^{2} T \frac{E_{b}^{q}}{2}+\eta_{q}
$$

where $E_{b}^{q}=2 \sum_{k \in \mathcal{K}}\left|\rho_{k}^{q}\right|^{2}$ is the energy of the transmitted symbol $q$. The term $\eta_{q}$ collects interference contributions from noise and interferer signals, but also collects cross-correlation of signal and noise disturbing the reference time slot and crosscorrelation of signal and noise disturbing the informationbearing time slot. An estimation of the transmitted binary data information is provided at the output of the decision circuit, such that $\hat{d}_{q}=\operatorname{sgn}\left(z_{q}\right)$, where $\operatorname{sgn}($.$) denotes the signum$ function of its argument. It is worth noting that robustness of this estimation is significantly improved by making use of the delay-and-sum beamforming technique. In equation (8) it is easy to verify that the interferences term $\eta_{q}$ is a function of the number of antennas $M$, when in the same time the term of interest (which contains $d_{q}$ ) is a function of $M^{2}$. Obviously, when $M$ increases, the term of interest becomes predominant regardless to the interferences term.

\section{DETECTION PERFORMANCE}

\section{A. Hypothesis}

In this section we are interested in the error probability of detection of the data information of a wideband signal. The direction of arrival $\theta$ of this wideband signal of interest (WSOI) is known. An additive white Gaussian noise is also collected by the array of antennas. This noise is assumed to be temporally and spatially white, and is present in the whole frequency band of the WSOI with a power spectral density of $N_{0}$. Belonging to the frequency band of the WSOI, a narrowband interferer (NBI) signal, having a bandwidth $W_{I}$, is impinging on the array with the angle of arrival $\theta_{I}$. This NBI signal is supposed to be a very high power signal with eventually a much higher symbol rate than the WSOI. Consequently, using the Fourier analyzer approach [10], models of the noise and the NBI signal can be given 
considering the relatively large symbol duration $T$ of the WSOI. Thus, a complete expression of the delay-and-sum output signal is

$$
\begin{aligned}
& y(t)=\sum_{q=0}^{\infty}\left(M \sum_{k \in \mathcal{K}} \rho_{k}^{q} g_{d_{q}}(t-2 q T) e^{j \omega k t}\right. \\
& +\sum_{m=1}^{M} \sum_{k \in \mathcal{K}} n_{m, k, 1}^{q} r(t-2 q T) e^{j \omega k\left(t-\tau_{m}(\theta)\right)} \\
& +\sum_{m=1}^{M} \sum_{k \in \mathcal{K}} n_{m, k, 2}^{q} r(t-(2 q+1) T) e^{j \omega k\left(t-\tau_{m}(\theta)\right)} \\
& +\sum_{m=1}^{M} \sum_{k \in \mathcal{K}_{\mathcal{I}}} \nu_{k, 1}^{q} r(t-2 q T) e^{j \omega k\left(t-\tau_{m}(\theta)+\tau_{m}\left(\theta_{I}\right)\right)} \\
& \left.+\sum_{m=1}^{M} \sum_{k \in \mathcal{K}_{\mathcal{I}}} \nu_{k, 2}^{q} r(t-(2 q+1) T) e^{j \omega k\left(t-\tau_{m}(\theta)+\tau_{m}\left(\theta_{I}\right)\right)}\right)
\end{aligned}
$$

with

$$
r(t)= \begin{cases}1 & \text { if } 0 \leq t<T \\ 0 & \text { elsewhere }\end{cases}
$$

In (9), $n_{m, k, i}^{q}(i=1,2)$ are the $k^{\text {th }}$ complex Fourier coefficients of the noise concerning the $i^{\text {th }}$ time slot of the $q^{\text {th }}$ symbol duration of the WSOI, at the $m^{t h}$ antenna. These coefficients are independent zero-mean complex circular Gaussian random variables, with the variance $\sigma_{n}^{2}=N_{0}$. Second order moments are then the followings

$$
\mathrm{E}\left[n_{m, k, i}^{q}\left(n_{m^{\prime}, l, j}^{p}\right)^{*}\right]=\sigma_{n}^{2} \delta_{p, q} \delta_{m, m^{\prime}} \delta_{k, l} \delta_{i, j}
$$

where $\delta_{i, j}$ is the Kronecker's delta. The spectral band of the NBI signal lying in the wide frequency band of the WSOI, it comes that $\mathcal{K}_{\mathcal{I}}$ is a subset of $\mathcal{K}$. In $(9), \nu_{k, 1}^{q}(i=1,2)$ are then the $k^{t h}$ complex Fourier coefficients of the NBI signal concerning the $i^{t h}$ time slot of the $q^{t h}$ symbol duration of the WSOI. In the following, these coefficients are assumed to be independent zero-mean complex circular random variables, with the variance $\sigma_{\nu}^{2}$. Second order moments are given by:

$$
\mathrm{E}\left[\nu_{k, i}^{q}\left(\nu_{l, j}^{p}\right)^{*}\right]=\sigma_{\nu}^{2} \delta_{p, q} \delta_{k, l} \delta_{i, j}
$$

By considering that this NBI signal is continuously transmitting, the mean-NBI-signal-energy captured during the WSOIsymbol duration $2 T$ is

$$
E_{I}=2 W_{I} T \sigma_{\nu}^{2}
$$

According to the delay-and-sum receiver and correlation-based detector structure given in figure 1 , a decision variable $z_{q}$ is provided which is the real part of the output of the correlation of the reference signal and the information-bearing signal of the $q^{\text {th }}$ transmitted symbol. It comes that this decision variable $z_{q}(7)$ is given by

$$
z_{q}=d_{q} M^{2} T \sum_{k \in \mathcal{K}}\left|\rho_{k}^{q}\right|^{2}+\eta_{q}
$$

where

$$
\begin{aligned}
& \frac{\eta_{q}}{T}=M \sum_{m=1}^{M} \sum_{k \in \mathcal{K}} \mathcal{R}\left\{\begin{array}{c}
d_{q} \rho_{k}^{q} n_{m, k, 1}^{q *} e^{j \omega k \tau_{m}(\theta)} \\
+\rho_{k}^{q *} n_{m, k, 2}^{q} e^{-j \omega k \tau_{m}(\theta)}
\end{array}\right\} \\
& +M \sum_{m=1}^{M} \sum_{k \in \mathcal{K}_{\mathcal{I}}} \mathcal{R}\left\{\begin{array}{l}
d_{q} \rho_{k}^{q} \nu_{k, 1}^{q *} e^{j \omega k \Delta_{m}} \\
+\rho_{k}^{q *} \nu_{k, 2}^{q} e^{-j \omega k \Delta_{m}}
\end{array}\right\} \\
& +\sum_{m, n=1}^{M} \sum_{k \in \mathcal{K}_{\mathcal{I}}} \mathcal{R}\left\{\begin{array}{l}
n_{m, k, 2}^{q} \nu_{k, 1}^{q *} e^{j \omega k\left(\Delta_{n}-\tau_{m}(\theta)\right)} \\
+n_{m, k, 1}^{q *} \nu_{k, 2}^{q} e^{-j \omega k\left(\Delta_{n}-\tau_{m}(\theta)\right)}
\end{array}\right\} \\
& +\sum_{m, n=1}^{M} \sum_{k \in \mathcal{K}_{\mathcal{I}}} \mathcal{R}\left\{\nu_{k, 2}^{q} \nu_{k, 1}^{q *} e^{j \omega k\left(\Delta_{m}-\Delta_{n}\right)}\right\} \\
& +\sum_{m, n=1}^{M} \sum_{k \in \mathcal{K}} \mathcal{R}\left\{n_{m, k, 2}^{q} n_{m, k, 1}^{q *}\right\}
\end{aligned}
$$

with $\Delta_{m}=\tau_{m}(\theta)-\tau_{m}\left(\theta_{I}\right)$.

\section{B. BER derivation}

The number of terms in (14) is very large and we may then approximate the decision variable $z_{q}$ as a normally distributed random variable, using the central limit theorem. Assuming that the data information $d_{q}$ can be +1 or -1 equiprobably, and arbitrarily setting $d_{q}=+1$, then an error occurs when $z_{q}$ is negative. The error probability, or Bit-Error-Rate (BER), is given by

$$
\mathrm{BER}=\frac{1}{2} \operatorname{erfc}\left(\frac{\mathrm{E}\left[z_{q}\right]}{\sqrt{2 \operatorname{Var}\left[z_{q}\right]}}\right)_{d_{q}=+1}
$$

Expectation of the decision variable is given by $\mathrm{E}\left[z_{q}\right]=$ $d_{q} M^{2} T \frac{E_{b}}{2}$. Recalling the statistical independence between random variables $\rho_{k}^{q}, n_{m, k, 1}^{q}, n_{m, k, 2}^{q}, \nu_{k, 1}^{q}$ and $\nu_{k, 2}^{q}$ in equation (14), it comes that the variance of the decision variable is given by

$$
\operatorname{Var}\left[z_{q}\right]=M^{4} T^{2} \operatorname{Var}\left[\sum_{k \in \mathcal{K}}\left|\rho_{k}^{q}\right|^{2}\right]+\operatorname{Var}\left[\eta_{q}\right]
$$

Expression of the variance $\operatorname{Var}\left[\sum_{k \in \mathcal{K}}\left|\rho_{k}^{q}\right|^{2}\right]$ is given by

$$
\operatorname{Var}\left[\sum_{k \in \mathcal{K}}\left|\rho_{k}^{q}\right|^{2}\right]=W T(W T+\Psi-1) \sigma_{\rho}^{4}-\frac{E_{b}^{2}}{4}
$$

where $\Psi$ is depending on the statistics of $\rho_{k}^{q}$ (see Appendix A). Derivation of the expression of $\operatorname{Var}\left[\eta_{q}\right]$ is quite easy to obtain, and finally a BER performance expression of the antenna array TR receiver system is the following

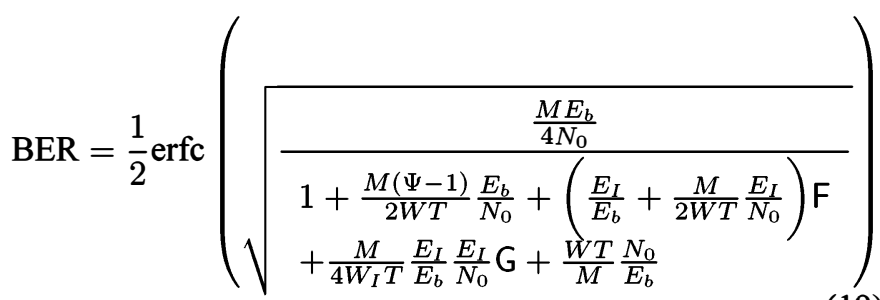

with factors $F$ and $G$ defined as

$$
\mathrm{F}=\frac{1}{M^{2} W_{I} T} \sum_{m, n=1}^{M} \sum_{k \in \mathcal{K}_{\mathcal{I}}} \cos \left(\omega k\left(\Delta_{m}-\Delta_{n}\right)\right)
$$

and

$\mathrm{G}=\frac{1}{M^{4} W_{I} T} \sum_{m, n, m^{\prime}, n^{\prime}=1}^{M} \sum_{k \in \mathcal{K}_{\mathcal{I}}} \cos \left(\omega k\left(\Delta_{m}-\Delta_{n}-\Delta_{m^{\prime}}+\Delta_{n^{\prime}}\right)\right)$

Factors $F$ and $G$ denote the NBI signal rejection (or cancellation) rate of the antenna array. These rejection rates depend on the difference of the directions of arrival between the WSOI and the NBI, but $F$ and $G$ also depend on the antenna array features (number of antennas, array topology...). When angles of arrival are equal $\theta=\theta_{I}$, factors are maximum $\mathrm{F}=\mathrm{G}=1$, and in this case disturbance caused by the NBI is maximum (no rejection). In a more general case when DOA of sources are different, theses factors are less than unity and can also equal zero in some cases (total rejection of the NBI signal). 


\section{Numerical results}

In this section, numerical results are provided in order to illustrate the performance improvement of a TR system by using an antenna array instead of a single antenna in presence of narrowband interference. Depending on the application, the $E_{b} / N_{0}$ ratio is not always the most significant feature, especially in some UWB low power consumption and low data rate communication cases, where the spectral efficiency is not as important as in high power and high rate systems. Thus, in the UWB framework, it seems more relevant to show the effect of the signal-to-noise ratio, or even better the signal-to-noise power-spectral-density ratio, on the BER performance of the system. This expression can be derived straightforwardly from (19), by defining $\gamma=\frac{\sigma_{\rho}^{2}}{N_{0}}$ the mean WSOI-to-noise powerspectral-density ratio (WSNR) in the frequency band $W$ of the WSOI, and $\zeta=\frac{\sigma_{\rho}^{2}}{\sigma^{2}}$ the mean WSOI-to-NBI power-spectraldensity ratio (WSIK) in the frequency band $W_{I}$ of the NBI. In the following simulations, the WSOI is considering to have a bandwidth of $W=2 \mathrm{GHz}$ from $2 \mathrm{GHz}$ to $4 \mathrm{GHz}$. A TR-based modulation is used and the half symbol duration $T=500 \mathrm{~ns}$; it comes that $W T=1000$. Let consider that the NBI signal is centered at frequency $2.4 \mathrm{GHz}$ with a narrow bandwidth of $W_{I}=10 \mathrm{MHz}$. At the receiver, the antenna array is a planar rectangular array of $M$ antennas, i.e., this array has a rectangular configuration of $N_{x} \times N_{y}=M$ elements, which are uniformly spared with a spacing of $5 \mathrm{~cm}$ one between another. In figure 2 , simulations have been conducted to be

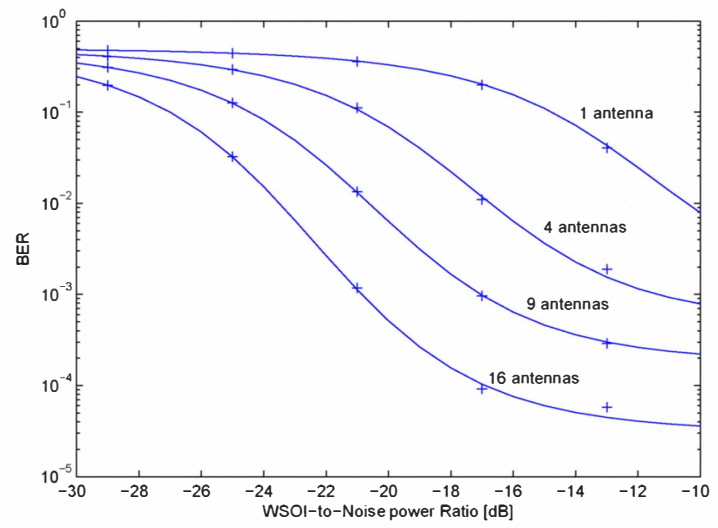

Figure 2. Theoretical BER (solid line) and computed BER (cross marks) versus WSNR $\gamma$; square antenna array of $1,4,9$ and 16 elements; WSIR $\zeta=-23 \mathrm{~dB}$

compared with the theoretical BER results. The BER is plotted when the WSNR $\gamma$ is varied, from $-30 \mathrm{~dB}$ to $-10 \mathrm{~dB}$. The NBI is present, and the DOA separation between the WSOI and the NBI is $10^{\circ}$. The WSIR is set to $\zeta=-23 \mathrm{~dB}$, thus the total mean power of the NBI is equal to the total mean power of the WSOI, i.e., $\frac{W}{W_{I}} \zeta=1$. Simulations are done with square array configurations $\left(N_{x}=N_{y}\right)$ of $M=1,4$, 9 and 16 antennas. Thus, when a single antenna is used the performance is poor. Involving multiple antennas allows to improve significantly the BER. Curves in figure 2 haven't got a classical waterfall shape but seems to be limited when $\gamma$ increases, this is due to the fact of the NBI power. When $\gamma=$
$-20 \mathrm{~dB}$, the power level of the WSOI is very low compared to the noise power level, and the delay-and-sum beamforming allows to increase this signal-to-noise power ratio so that the demodulation is improved. When, $\gamma=-10 \mathrm{~dB}$, the power level of the WSOI is still below the noise power level, but the main perturbation is coming from the NBI, recalling that $\zeta=-23 \mathrm{~dB}$. The more the antenna array is large, the more it has a high rejection power. The capacity of the delay-andsum beamforming to cancel interferences depends mainly on the array configuration and on the DOA separation between incoming signals.

In order to characterize the rejection of the NBI by an

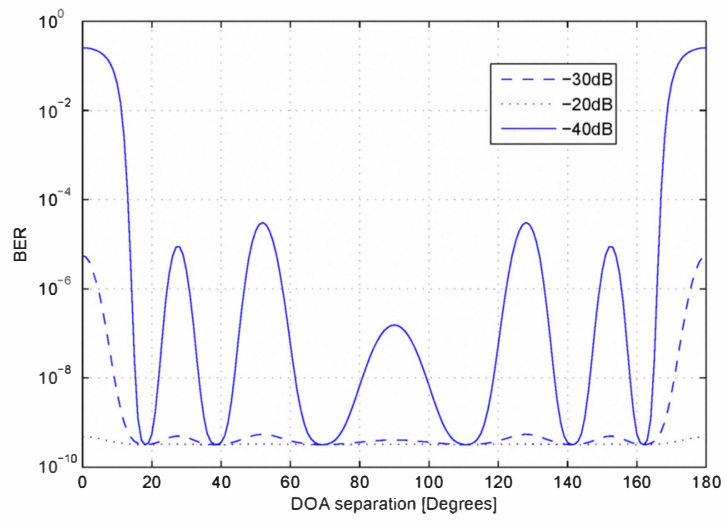

Figure 3. BER versus the DOA separation; with different values of WSIR $\zeta=-20,-30,-40 \mathrm{~dB}$; WSNR $\gamma=-20 \mathrm{~dB}$

antenna array, BER performance are provided in figure 3 when the angular separation with the WSOI is varied and when the array has a $2 \times 8$ rectangular configuration. The WSNR is set to $\gamma=-20 \mathrm{~dB}$, and several values of the WSIR $\zeta$ are chosen $(-20 \mathrm{~dB},-30 \mathrm{~dB}$ and $-40 \mathrm{~dB})$. In this figure, it appears clearly that for some given DOA separations, which are directly associated to the array configuration, the NBI power is canceled. For these DOA separation values, the BER is minimum and is obviously equal for any $\zeta$. Thus, the antenna array allows complete cancellation of the NBI power at the output of the delay-and-sum part of the receiver, it could be observed that the $F$ and $G$ factors are equal to zero for such DOA separations. On the other hand, the rejection of the NBI is less efficient for some other values of the DOA separation. Shapes of the BER curves in figure 3 can be easily connected to that of the gain pattern of the rectangular array of $2 \times 8$ antennas around the center frequency of the narrowband $W_{I}$. A perfect symmetry of the BER curves relatively to the DOA separation $90^{\circ}$ can be observed in the figure. This behavior comes from the fact that the array geometry itself exhibits some symmetries, which force the array gain or radiation pattern into ambiguity features.

When now a linear uniform array of $M$ elements is used (i.e., a $1 \times M$ antenna array), BER is plotted versus the DOA separation, by varying the length of the antenna array in order to observe the dependency of the rejection power to the array configuration. In figure 4 , the WSNR is $\gamma=-20 \mathrm{~dB}$, the WSIR is $\zeta=-30 \mathrm{~dB}$, several values of $M$ are chosen and 




Figure 4. BER versus the DOA separation; with linear arrays of $6,8,10$ and 12 antennas; WSNR $\gamma=-20 \mathrm{~dB}$; WSIR $\zeta=-30 \mathrm{~dB}$

the DOA separation is varied for $0^{\circ}$ to $180^{\circ}$. In the former figure, it can be observed that when DOA of the WSOI and that of the NBI are equal (DOA separation of $0^{\circ}$ ), the system is in the worst case. There is no benefit to use an antenna array in such a condition because the rejection power of the array is not involved ( $F$ and $G$ factors are equal to unity). But, by increasing $M$ the size of the array, the BER performance is improved when the DOA separation is not zero, and BER curves look like a radiation pattern, with a main lobe centered at $0^{\circ}$ and some lower side lobes. When $M$ increases, the mainlobe width of such a pattern decreases and the side-lobe levels decrease too, i.e., the NBI power rejection is improved. This is obviously due to the array radiation pattern at the frequency of the incoming NBI signal, which exhibits a main lobe and some side lobes.

\section{CONCLUSION}

In this paper, an analysis of the improvement of the BER performance for TR-based UWB communication systems has been investigated, in presence of narrowband interference. By using an array of antennas at the receiver and performing a delay-and-sum beamforming, it has been shown how the performance can be drastically increased without adding any other active and power-consuming filters. A wideband TR signal of interest and a narrowband interferer signal have been considered at the input of an antenna array receiver. The delay-and-sum beamforming structure acts as a spatial filter. An analytical BER expression has been derived, showing that the performance depends on many parameters, mainly the frequency bandwidth of the signals, the signal-to-noise power ratio, the power of the narrowband interference, and obviously the antenna array characteristics (number of antennas, geometry,...). This study has been conducted according to a generalized model of communication waveforms, so that it is valid for any kind of carrier waveforms,i.e., fixed impulses or chaotic waveforms. Finally, the proposed analysis problem has to be further investigated by considering multipath channels as it is often encountered and, by considering also the problem of the estimation errors of the DOA.

\section{APPENDIX}

Let observe that

$$
\mathrm{E}\left[\left(\sum_{k \in \mathcal{K}}\left|\rho_{k}^{q}\right|^{2}\right)^{2}\right]=W T \mathrm{E}\left[\left|\rho_{k}^{q}\right|^{4}\right]+2\left(\begin{array}{c}
W T \\
2
\end{array}\right) \sigma_{\rho}^{4}
$$

Then the following expansion can be given

$$
\mathrm{E}\left[\left|\rho_{k}^{q}\right|^{4}\right]=\mathrm{E}\left[\mathcal{R}\left\{\rho_{k}^{q}\right\}^{4}+\mathcal{I}\left\{\rho_{k}^{q}\right\}^{4}+2 \mathcal{R}\left\{\rho_{k}^{q}\right\}^{2} \mathcal{I}\left\{\rho_{k}^{q}\right\}^{2}\right]
$$

Let now recall the assumption concerning the fact that $\rho_{k}^{q}$ is a zero-mean complex circular random variable, having the variance $\sigma_{\rho}^{2}$. Hence, $\mathcal{R}\left\{\rho_{k}^{q}\right\}$ the real part and $\mathcal{I}\left\{\rho_{k}^{q}\right\}$ the imaginary part of $\rho_{k}^{q}$ are two zero-mean independent real random values with variance $\frac{\sigma_{\rho}^{2}}{2}$. Let now define arbitrarily (as an example) that these random values are uniformly distributed, then it comes that $\mathrm{E}\left[\mathcal{R}\left\{\rho_{k}^{q}\right\}^{4}\right]=\mathrm{E}\left[\mathcal{I}\left\{\rho_{k}^{q}\right\}^{4}\right]=\frac{9}{20} \sigma_{\rho}^{4}$ and finally that $\mathrm{E}\left[\left|\rho_{k}^{q}\right|^{4}\right]=\frac{7}{5} \sigma_{\rho}^{4}$. Then in a more general case, it can be established that

$$
\mathrm{E}\left[\left(\sum_{k \in \mathcal{K}}\left|\rho_{k}^{q}\right|^{2}\right)^{2}\right]=W T(W T+\Psi-1) \sigma_{\rho}^{4}
$$

where $\Psi=\frac{7}{5}$ in our example. Then, variance (18) can be given.

\section{REFERENCES}

[1] Y. Souilmi "Analysis of signaling and coding of non-coherent schemes for ultra-wideband systems," PhD Thesis, Institut Eurecom, Sophia-Antipolis, France, June 2005.

[2] T.Q.S. Quek, Q. Win "Analysis of UWB TR communication systems in dense multipath Channels," IEEE journal on selected areas in communications, vol. 23, no. 9, pp. 1863-1874, September 2005.

[3] H. Arslan, Z.N. Chen, M.G. Di Benedetto, "Ultra wideband Wireless Communication," John Wiley and Sons, 2006.

[4] L. Stoica, "Non-coherent energy detection transceivers for ultra-wideband impulse radio systems," PhD thesis, Faculty of technology of the university of Oulu, Finland, February 2008.

[5] M. Sushchik, L.S. Tsimring, A.R. Volkovskii, "Performance analysis of correlation-based communication schemes utilizing chaos," IEEE Transactions on Circuits and Systems I: Fundamental Theory and Applications, vol. 47, pp. 1684-1691, December 2000.

[6] Z. Zhou, T. Zhou, J. Wang, "Performance of Multiple-Access DCSK Communication over a Multipath Fading Channel with Delay Spread," Birkhäuser, Circuits Systems and Signal Processing, vol. 27, pp. 507-518, 2008.

[7] S. Kaza, "Performance analysis of Ultra-Wideband transmitted reference system and enhancement techniques," PhD Thesis of the Faculty of the Graduate School Tennessee Technological University, December 2004.

[8] X. Liao, S. Zhu, E. Zeng, "Multiple-Antenna Receiving and Frequency Domain Equalization in Transmitted-Reference UWB Systems," IEICE Transactions on Communications, vol. E91, no. 7, pp. 2405-2408, July 2008.

[9] Q. Zhang, A. Nallanathan, "Delay-Sum Antenna Array Reception for Transmitted-Reference Impulse Radio (TR-IR) Systems," IEEE Transactions on Wireless Communications, vol. 7, no. 12, pp. 5208-5213, December 2008.

[10] G. Kolumbán, F.C.M. Lau, C.K. Tse, "Generalization of waveform communications : The Fourier analyzer approach," Birkhäuser, Circuits Systems and Signal Processing, vol. 24, no. 5, pp. 451-474, 2005.

[11] G. Kolumbán, T. Krébesz, F.C.M. Lau, C.K. Tse, "A mathematical approach to derive optimum detector configurations for UWB radio applications," International Symposium on Nonlinear Theory and its Applications (NOLTA'08), Budapest, Hungary, September 2008. 